\title{
Development of the GEM-TPC X-ray Polarimeter with the Scalable Readout System
}

\author{
Takao Kitaguchi ${ }^{1,2, a}$, Asami Hayato ${ }^{2}$, Wataru Iwakiri², Yoko Takeuchi²,3, Megu Kubota ${ }^{2,3}$, Kazuki Nishida ${ }^{2,3}$, Teru- \\ aki Enoto ${ }^{4,2}$, and Toru Tamagawa ${ }^{2}$ \\ ${ }^{1}$ Department of Physical Sciences, Hiroshima University, Higashi-Hiroshima, Hiroshima 739-8526, Japan \\ ${ }^{2}$ RIKEN Nishina Center, 2-1 Hirosawa, Wako-shi, Saitama 351-0198, Japan \\ ${ }^{3}$ Department of Physics, Tokyo University of Science, 1-3 Kagurazaka, Shinjuku-ku, Tokyo 162-8602, Japan \\ ${ }^{4}$ Department of Astronomy, Kyoto University, Sakyo-ku, Kyoto 606-8502, Japan
}

\begin{abstract}
We have developed a gaseous Time Projection Chamber (TPC) containing a single-layered foil of a gas electron multiplier (GEM) to open up a new window on cosmic X-ray polarimetry in the $2-10 \mathrm{keV}$ band. The micro-pattern TPC polarimeter in combination with the Scalable Readout System produced by the RD51 collaboration has been built as an engineering model to optimize detector parameters and improve polarimeter sensitivity. The polarimeter was characterized with unpolarized X-rays from an X-ray generator in a laboratory and polarized X-rays on the BL32B2 beamline at the SPring- 8 synchrotron radiation facility. Preliminary results show that the polarimeter has a comparable modulation factor to a prototype of the flight one.
\end{abstract}

\section{Introduction}

Recent progress in micro-pattern gas detectors enables us to sensitively and shapely track charged particles including photoelectrons with an energy down to $1 \mathrm{keV}$. The angular distribution of photoelectrons originated from the photoelectron effect is sensitive to the electric field vector (or polarization direction) of incident X-rays. Since the photoelectron effect is the dominant interaction of X-rays, the micro-pattern gas polarimeter is expected to open up a new window on cosmic X-ray polarimetry.

So far, the Crab Nebula is the only astronomical source that has been detected by X-ray polarization measurements since the 1970s [1]. In order to make a breakthrough in cosmic X-ray polarimetry, three satellite missions, each of which will carry micro-pattern gas polarimeters utilizing the photoelectric effect in orbit, were selected in 2015 as a promising candidate for launch in the 2020s. XIPE (X-ray Imaging Polarimetry Explorer) [2] is an ESA medium-class mission candidate, while IXPE (Imaging Xray Polarimetry Explorer) and PRAXyS (Polarimeter for Relativistic Astrophysical X-ray Sources) [3] are NASA Small Explore finalists.

Micro-pattern gas polarimeters can detect the X-ray polarization by imaging a 2-D photoelectron track projected on to a plane perpendicular to the incident X-ray direction. They are mainly divide into two types according to the X-ray imaging capability. XIPE and IXPE will carry X-ray imaging polarimeters with a 2-D readout system $[4,5]$. PRAXyS will have a nonimaging but higher sensitive polarimeter with 1-D strip electrodes using the

\footnotetext{
${ }^{a}$ e-mail: kitaguti@hiroshima-u.ac.jp
}

time projection chamber (TPC) technique $[6,7]$. In addition, the electric drift field direction is different: the imaging polarimeter has parallel electric field to the incident $\mathrm{X}$-ray direction, whereas the nonimaging one has perpendicular field and therefore is unable to determine an interaction point along the electron drift direction. Instead, the latter polarimeter has more thicker absorption volume along the incident X-ray direction.

This paper deals with development of an engineering model of the micro-pattern TPC polarimeter combined with the Scalable Readout System (SRS) [8] produced by the RD51 Collaboration [9]. The polarimeter with SRS has been developed for ground tests to improve polarimetry sensitivity by quickly and flexibly changing detector parameters such as gas pressure and electric field strengths. Preliminary results of polarimeter performance evaluation is also described. However, more detailed results will be described elsewhere.

\section{Overview of X-ray polarimeter}

The detailed design and detection concept of the micropattern TPC polarimeter for cosmic X-rays onboard PRAXYS is described in [6]. A schematic view of the polarimeter with SRS is illustrated in Figure 1. The polarimeter chamber is sealed with dimethyl ether gas at low pressure of 190 Torr and consists of a thick $(8 \mathrm{~cm}$ along the incident $\mathrm{X}$-ray direction) drift/absorption region between the drift electrode and the gas electron multiplier (GEM) cathode and an induction region between the GEM anode and 128-ch readout strips with a pitch of $121 \mu \mathrm{m}$. These devices installed in the chamber for the engineering model 


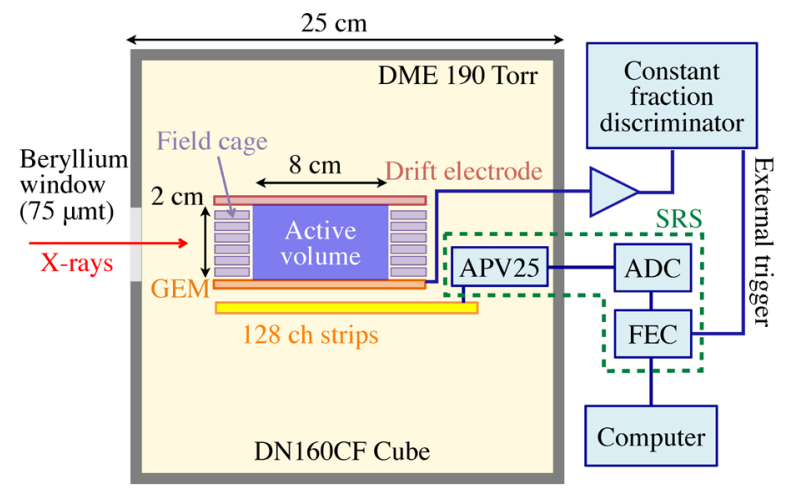

Figure 1. Schematic view of the polarimeter combined with SRS. The detector is self-triggered by using the GEM cathode signal in input to the trigger connector of SRS/FEC. The polarimeter chamber is placed on the $\mathrm{X}-\mathrm{Z}$ stage to adjust the $\mathrm{X}$-ray beam position.

Table 1. Difference of polarimeter parameters.

\begin{tabular}{lcc}
\hline \hline & \multicolumn{2}{c}{ Polarimeter } \\
& This work & Flight model \\
\hline Sampling number & 27 & 30 \\
Sampling rate $(\mathrm{MHz})$ & 40 & 20 \\
Drift field $(\mathrm{V} / \mathrm{cm})$ & 374 & 196 \\
Induction gap (mm) & 1 & 0.25 \\
\hline
\end{tabular}

are almost the same as those of the flight prototype. The main difference between them is a signal readout system: the flight prototype uses the APV25 ASIC [10] controlled by a NASA-programmed FPGA, whereas the polarimeter utilizes SRS with APV25. The other differences on detector settings are listed in Table 1. Because the ADC sampling rate of SRS is fixed at $40 \mathrm{MHz}$ and twice as fast as that of the flight polarimeter, the electron drift velocity is set to be $4.84 \mu \mathrm{m} \mathrm{ns}^{-1}$ by adjusting the drift field strength so that the TPC image pixel size is $121 \mu \mathrm{m}$ for both polarimeters.

An online data analysis software dedicated to X-ray polarimetry was developed based on the ROOT analysis framework [11]. The event-by-event analysis software consists of three threads: an event receiver, an analyzer/recorder, and a monitor. A quick-look monitor to display plots of a track image, spectrum, modulation curve, and so on as shown in Figure 2 allows us to check preliminary results of the polarization measurement as soon as data from SRS are taken. The data analyzer mainly performs track image processing which execute pedestal subtraction, common mode noise subtraction, electronics response deconvolution, and photoelectron angle reconstruction in sequence. Before X-ray beam irradiation, data of the pedestal and the impulsive electronics response are acquired with periodic internal triggers generated by FPGA on the SRS front-end card (FEC) and periodic test pulses from the on-chip calibration circuit on APV25, respectively.

\section{Preliminary results of polarimeter performance}

In order to characterize the polarimeter, it was irradiated with unpolarized X-rays in a laboratory and polarized Xrays on the BL32B2 beamline at the SPring-8 synchrotron facility. Unpolarized X-rays at energies of 4.5 and $6.4 \mathrm{keV}$ separately generated by an X-ray generator were used to calibrate polarimeter parameters (gas pressure, high voltages, image processing parameters, and so on) so that the modulation curve, which is a distribution of photoelectron emission angles, becomes flat. The parameters derived from the unpolarized data were then used for polarized data. Polarized and collimated X-rays were generated at four energies $(4.5,5.5,6.4$, and $7.5 \mathrm{keV})$ at five detector positions or drift lengths from the GEM cathode (4.6, 7.6, 10.6, 13.6 and $16.6 \mathrm{~mm})$. The polarized beam test at SPring-8 was performed in December 7-15, 2014.

Figure 3 shows $6.4 \mathrm{keV}$ spectra at various detector positions. The energy resolution (FWHM) of all the spectra is $22 \%$, which is larger than that of a prototype of the flight polarimeter, $16 \%$ [12]. The broader energy resolution is due to a non-uniform gain of the GEM foil [13] and could be improved by replacing it with a foil of improved quality. The spectral peak is shifted to the lower channel with the increase of the drift distance because of electron attachment of impurity gas caused by outgassing [14]. An electron attachment coefficient at the drift field of $374 \mathrm{~V} \mathrm{~cm}^{-1}$ is $\sim 0.05 \mathrm{~cm}^{-1}$, derived by exponential fitting to the spectral peak as a function of the drift distance. The spectral peak shift due to electron attachment (not seen in the flight equivalent polarimeter [12]), can be reduced by long-term bake-out, which was performed, for the polarimeter chamber, with the temperature of $80^{\circ} \mathrm{C}$ for a week before the beam test.

Figure 4 shows photoelectron tracks with various energies at different X-ray irradiation positions. Some of them are curved due to large-angle Coulomb scattering with DME gas. The pixel region having a large amount of charge is the Bragg peak which represents the end point of the track. The track length increases with the increase of the incident X-ray energy. In addition, the track is more blurred with the increase of the electron drift length due to electron diffusion. The diffusion size for $1 \mathrm{~cm}$ drift length at the electric field of $374 \mathrm{~V} \mathrm{~cm}^{-1}$ is $140 \mu \mathrm{m}$ or 1.2 pixel, which is numerically calculated with Magboltz [15].

In order to reconstruct the photoelectron emission angle, the major principal axis with the origin at the barycenter from the charge distribution of the track image is regarded as the reconstructed angle. The Bragg peak, which represents the track end point and is unnecessary to reconstruct the initial angle of photoelectron emission, is clearly seen in long and curved tracks with a high eccentricity (e.g. the top left panels of Figure 4). Therefore, for such high eccentricity tracks, the half of the charge distribution which is separated by the minor principal axis and contains the Bragg peak is cut off. Then, the major principal axis, which is regarded as the revised photoelectron emission angle, is calculated again with the remaining charge distribution. The Bragg peak cut improves the modulation 

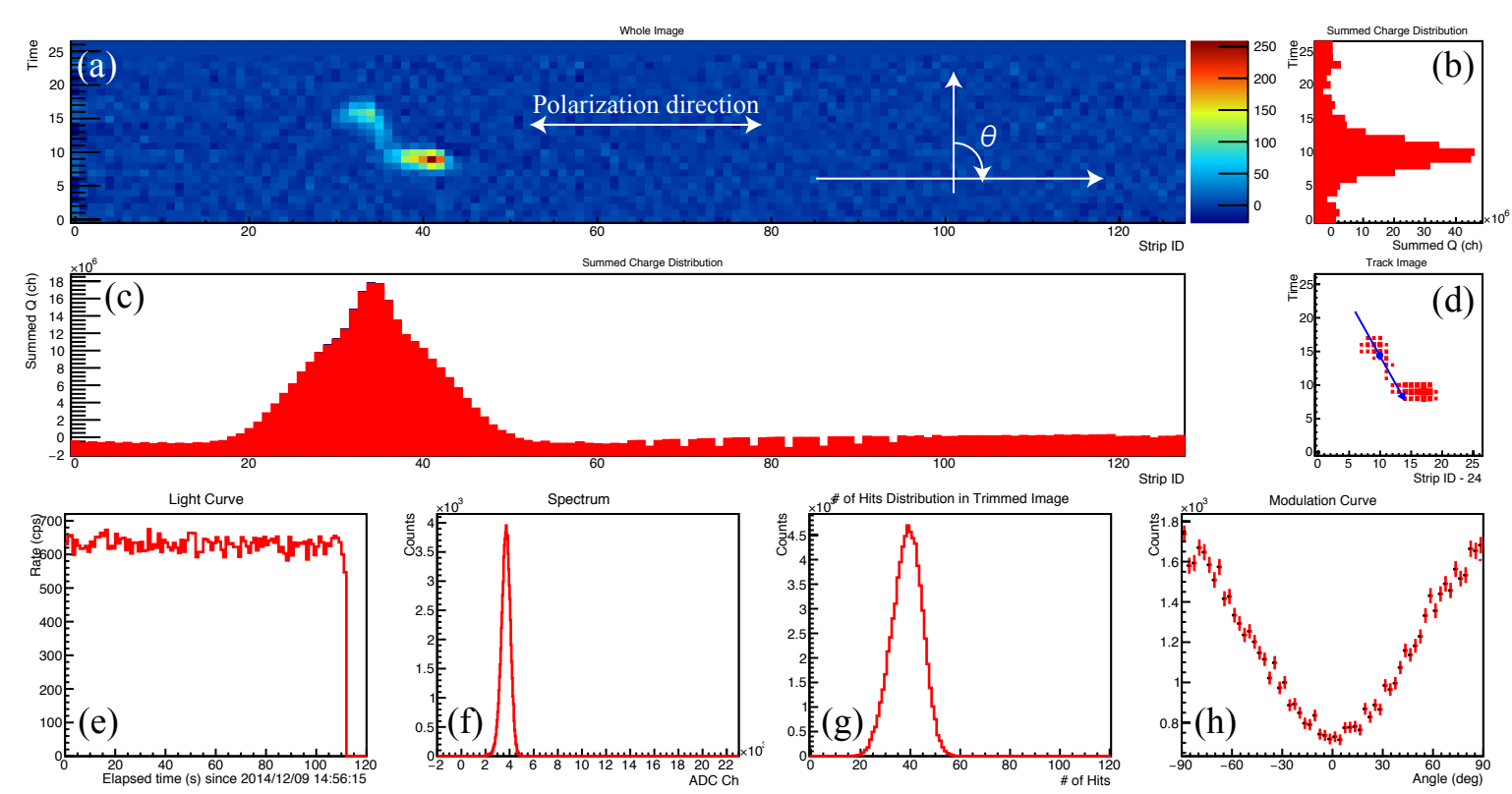

Figure 2. Online monitor to display polarization measurement data. Individual plots represent (a) full ( $27 \times 128$ pixels) track image with the $\theta$ angle definition, (b) and (c) stacked image projected to the Y-and X-axis of the track image, respectively, (d) trimmed $(27 \times 27$ pixels) track image centering on the charge centroid with the blue arrow representing a estimated angle of photoelectron emission direction, (e) time variation of count rates, (f) ADC channel spectrum, (g) distribution of the number of hit pixels, and (h) modulation curve in the coordinate system that 0 deg points the top of the track image. The data were taken by irradiation of polarized X-rays with the energy of $6.4 \mathrm{keV}$ at SPring-8/BL32B2.

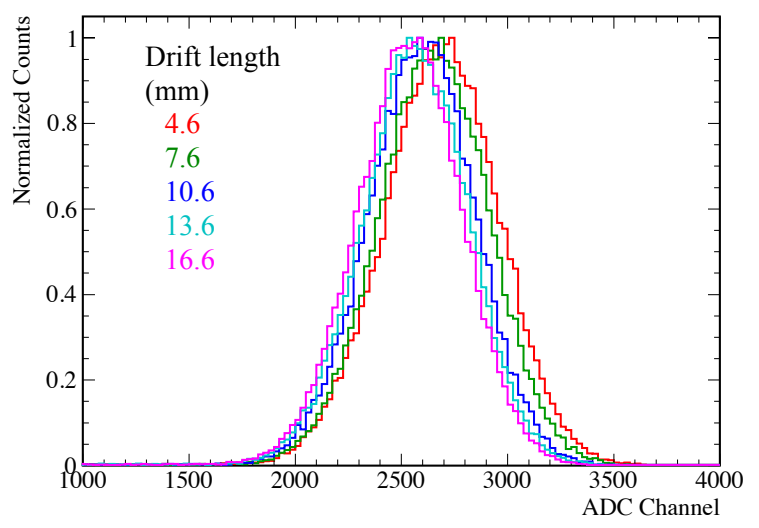

Figure 3. Measured spectra with $6.4 \mathrm{keV}$ X-ray irradiation at different detector positions. The ADC channel of each event is total amount of charge in the trimmed track image with the size of $27 \times 27$ pixels.

factor of high-energy ( $>5 \mathrm{keV}$ ) X-rays by approximately $10 \%$. A preliminary result of the modulation factor for $6.4 \mathrm{keV} \mathrm{X}$-rays at $7.6 \mathrm{~mm}$ drift length, which is near the optical axis of the polarimeter combined with an X-ray mirror, is approximately $50 \%$, comparable to that of the flight equivalent polarimeter [12].

\section{Summary}

The engineering model of the micro-pattern TPC polarimeter for cosmic X-rays has been developed in com- bination with the SRS/AVP25 signal readout module for ground tests. The polarimeter was characterized with polarized X-rays at SPring-8/BL32B2. Preliminary results show that the developed polarimeter has a comparable modulation factor to the flight equivalent one.

\section{Acknowledgements}

The synchrotron radiation experiments were performed at the BL32B2 of SPring-8. We would like to thank Dr. Dai Takei and Dr. Yuki Takayama for their supports on X-ray beam adjustment. T.K. was supported by JSPS Grant-in-Aid for Young Scientists (B) (No. 24740185). This work was partially supported by MEXT KAKENHI Grant Number 24105007.

\section{References}

[1] M.C. Weisskopf, G.G. Cohen, H.L. Kestenbaum, K.S. Long, R. Novick, R.S. Wolff, Astrophys. J. Lett. 208, L125 (1976)

[2] P. Soffitta, X. Barcons, R. Bellazzini, J. Braga, E. Costa, G.W. Fraser, S. Gburek, J. Huovelin, G. Matt, M. Pearce et al., Experimental Astronomy 36, 523 (2013)

[3] K.M. Jahoda, J.K. Black, J.E. Hill, T.R. Kallman, P.E. Kaaret, C.B. Markwardt, T. Okajima, R. Petre, Y. Soong, T.E. Strohmayer et al., X-ray polarization capabilities of a small explorer mission, in SPIE Conference Series (2014), Vol. 9144, p. 0

[4] E. Costa, P. Soffitta, R. Bellazzini, A. Brez, N. Lumb, G. Spandre, Nature 411, 662 (2001) 


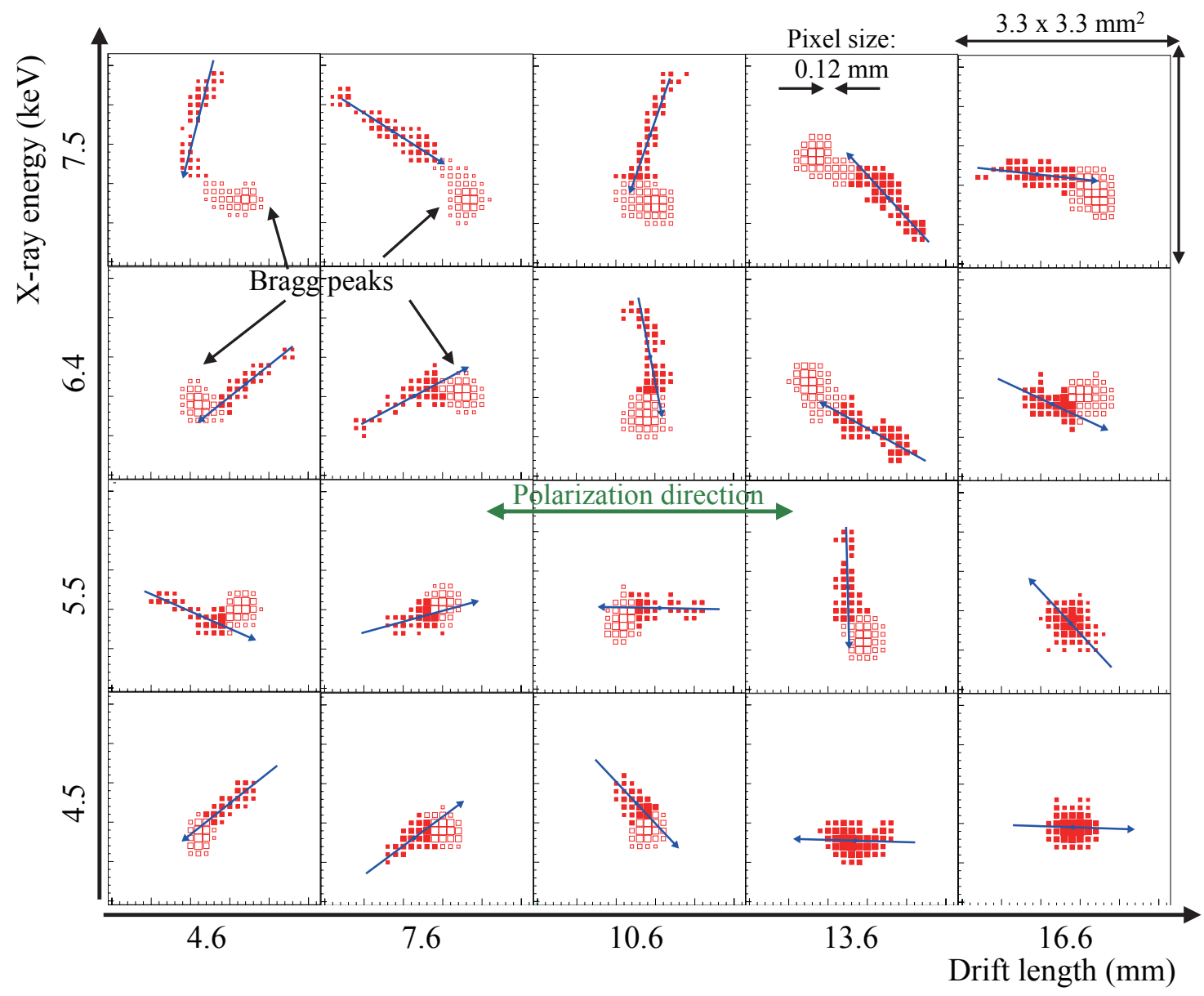

Figure 4. Zoo of photoelectron track images acquired by the polarimeter with SRS. Although the full image size is 128 (the number of strip electrodes) $\times 27$ (sampling number), the above images are trimmed to a square image with a size of $27 \times 27$ centering on the charge centroid. The red box area is proportional to charge amount. The open red boxes around the Bragg peaks show unused pixels for angle reconstruction of photoelectron emission.

[5] R. Bellazzini, G. Spandre, M. Minuti, L. Baldini, A. Brez, L. Latronico, N. Omodei, M. Razzano, M.M. Massai, M. Pesce-Rollins et al., Nuclear Instruments and Methods in Physics Research A 579, 853 (2007)

[6] J.K. Black, R.G. Baker, P. Deines-Jones, J.E. Hill, K. Jahoda, Nuclear Instruments and Methods in Physics Research A 581, 755 (2007)

[7] J.E. Hill, J.K. Black, T.J. Emmett, T. Enoto, K.M. Jahoda, P. Kaaret, D.S. Nolan, T. Tamagawa, Design improvements and $x$-ray performance of a time projection chamber polarimeter for persistent astronomical sources, in SPIE Conference Series (2014), Vol. 9144 , p. 1

[8] S. Martoiu, H. Muller, A. Tarazona, J. Toledo, Journal of Instrumentation 8, C3015 (2013)

[9] S. Duarte Pinto (RD51), Micropattern gas detector technologies and applications, the work of the RD51 collaboration, in Proceedings, 2010 IEEE Nuclear Science Symposium and Medical Imaging Conference (NSS/MIC 2010) (2010), pp. 802-807

[10] M.J. French, L.L. Jones, Q. Morrissey, A. Neviani, R. Turchetta, J. Fulcher, G. Hall, E. Noah, M. Ray- mond, G. Cervelli et al., Nuclear Instruments and Methods in Physics Research A 466, 359 (2001)

[11] R. Brun, F. Rademakers, Nuclear Instruments and Methods in Physics Research A 389, 81 (1997)

[12] T. Enoto, J.K. Black, T. Kitaguchi, A. Hayato, J.E. Hill, K. Jahoda, T. Tamagawa, K. Kaneko, Y. Takeuchi, A. Yoshikawa et al., Performance verification of the Gravity and Extreme Magnetism Small explorer (GEMS) x-ray polarimeter, in SPIE Conference Series (2014), Vol. 9144, p. 4

[13] Y. Takeuchi, T. Kitaguchi, A. Hayato, T. Tamagawa, W. Iwakiri, F. Asami, A. Yoshikawa, K. Kaneko, T. Enoto, K. Black et al., Properties of the flight model gas electron multiplier for the GEMS mission, in SPIE Conference Series (2014), Vol. 9144, p. 4

[14] J.E. Hill, J.K. Black, L. Brieda, P.L. Dickens, K. Montt de Garcia, D.L. Hawk, A. Hayato, K. Jahoda, J. Mohammed, Lifetime estimation of a time projection chamber $x$-ray polarimeter, in SPIE Conference Series (2013), Vol. 8859, p. 0

[15] S.F. Biagi, Nuclear Instruments and Methods in Physics Research A 421, 234 (1999) 